\title{
Traditional Uses of Medicinal Plants in Their Conservation in Charmang Village, Bajaur, KP, Pakistan
}

\author{
Syed Abidullah ${ }^{1, ~}$, Abdur Rauf ${ }^{1}$, Shah Wali Khan ${ }^{1}$, Mehnaz $^{1}$, Aisha Bibi ${ }^{2}$, Abdur Rauf ${ }^{1}$, \\ Sommya Fazal ${ }^{3}$ \\ ${ }^{1}$ Department of Botany, Abdul Wali Khan University, Mardan, Pakistan \\ ${ }^{2}$ Department of Biochemistry, Shaheed Benazir Bhutto Women, University, Peshawar, Pakistan \\ ${ }^{3}$ Department of Physics, Shaheed Benazir Bhutto Women University, Peshawar, Pakistan \\ Email address: \\ rauf77@awkum.edu.pk (A. Rauf), shahawkum44@yahoo.com (S. W. Khan), waqarali1464@gmail.com (Mehnaz), \\ eshafazal25@gmail.com (A. Bibi), araufpk476@gmail.com(A. Rauf),sommyafazal@gmail.com (S. Fazal), \\ charmang5@gmail.com (S. Abidullah) \\ *Corresponding author
}

\section{To cite this article:}

Syed Abidullah, Abdur Rauf, Shaha Wali Khan, Mehnaz, Aisha Bibi, Abdur Rauf, Sommya Fazal. Traditional Uses of Medicinal Plants in Their Conservation in Charmang Village, Bajaur, KP, Pakistan. International Journal of Bioorganic Chemistry.

Vol. 4, No. 1, 2019, pp. 70-83. doi: 10.11648/j.jibc.20190401.20

Received: February 10, 2019; Accepted: May 10, 2019; Published: June 4, 2019

\begin{abstract}
The Ethno-medicinal studies have been carried out for the first time in Charmang, Tehsil Nawagai, district Bajaur, Khyber Pakhtunkhwa (KP), Pakistan. In this project a total of 64 medicinal plants belonging to 39 families and 58 genera and species have been documented, which are being used by the local communities for the treatment of different human sicknesses. Among 64 plant species, 58 medicinal plants species were found as compared with the 12 earlier published articles. This study mainly focused to protect the world's cultural tradition and to document the importance of medicinal plants present in the research area. A complete data has been established on the general and worldwide uses of the projected study to find out new possible species for further studies. A general field surveys was arranged in the desire area, which was based on semistructured meetings of 135 local sneaks. To assess the exact herbal knowledge, the data were investigated using quantifiable Ethno medicinal records as RFC (Relative Frequency Citation) and UV (Use Value), separately. The local interviewed peoples used 64 plant species belonging to 58 genera in 39 families, mainly Lamiaceae, Rhamnaceae and Solanaceae. The type of most common diseases $(25 \%)$ was Respiratory diseases (Asthma, Throat infection, Cough etc.). The present study was emphasized based on ironic herbal knowledge to know the novel findings of medicinal plants in district Bajaur, KP, Pakistan which could be a standard use for upgrading the knowledge in terms of plant phytochemistry, pharmacology and biological activities for the efficiency and safety of medicinal plants.
\end{abstract}

Keywords: Ethnobotany, Medicinal Plants, Charmang, Bajaur, Pakistan

\section{Introduction}

Ethnobotany is old as human civilization on earth [1]. Most of the peoples use these plants for different purposes like nutrition and medicine since the establishment of these civilizations [2]. In these modern years the Ethnobotanical studies have grown motivation at global level to make sure that plants are used for the treatment of different diseases, medicines and natural drugs are discovering day by day for the treatment of diseases [3]. In the local communities the traditional information almost, the practice of medicinal plants is transferred from generation to generation [4], although the plants have traditionally helped as most important weapon against pathogen in man [5]. Medicinal plants are widely used almost in every community either directly or indirectly in current medicine system [6]. From earliest times some numbers of medicinal plants are being used for treating many sicknesses. The homoeopathic plant information and their practices by local Hakeem's and medicine development are not only treasured for management of ethnic practices and biodiversity but also for 
community health care [7]. Pakistan as a developing country more than $75 \%$ of citizens in different areas still rely on traditional medications as primary health care $[8,9]$. In this modern life style, the societies are going for away from the knowledge about the local medicinal plants is going to disappear among younger ones [3]. Today is the time to document this prosperity of knowledge about the natural medicinal plants for next generations throughout the world generally and specially in Pakistan particularly [3]. Plant species play a significance role in modern medicine, forming and pharmaceutical application [10]. Around 25\% drugs are produced directly from the plants and many other drugs are synthetic from the raw compounds which are isolated from plants. Around $80 \%$ of the people of developing countries are still dependent on traditional indigenous medicines for their basic health care [1]. In Pakistan out of 6000 flowering plants about 372 species are very common, out of these species 200 plants species are used as a medicine by local societies [1]. In 1987, Pie and Manandhara reported that $70 \%$ to $80 \%$ of the Himalayan range's community depends on medicinal plants of the local area [11]. People of the mountains area of Pakistan use plants for medicines, fire wood, food, fodder and timber wood [12]. The medically important plants of Himalayas are detailed from region to region and their distribution is dispersed and limited to small areas [1]. Plants are more important for us as compared to animals, due to their various collection of Rashid biochemical with a variety of potent biotic activities which is use in traditional healing systems [13]. In local people, different modes of application have been adopted to deed the natural flora [14]. In many rural communities, use of wild flora for medicine and food is banned since times [15]. Pakistan is full of medicinal plants that are used by rural people for a variety of purposes [1618]. Ethnobotany of different areas of Pakistan has been reported by different researchers $[1,19]$. According to World health organization (WHO) around $80 \%$ of the world's human population depends on herbal medications for their crucial health care system (World Health Organization, 2003) [20]. In the last periods in Pakistan, important technical and profitable tendencies have been experiential due to the common cultural suitability and commercial budding of plants founded Crops [18]. Pakistan is situated at altitude of $70.40^{\circ}-70.30^{\circ} \mathrm{N}$ and the longitude of $32.48^{\circ}-33.23^{\circ} \mathrm{E}$. It is situated at $340 \mathrm{~m}$ above the sea level.

The proposed study area i. e. Charmang tehsil Nawagai, spreads $22 \mathrm{~km}^{2}$ and is surrounded by Chamar khand, Kunar province from Afghanistan, Nakhter, Khamer Sar and Loesum. The total rural population of the Nawagai tehsil is about 79002 and the total House Hold population is 7,649 (www.pbscensus.gov.pk). It comprised of mountainous land with variety of significant medicinal plants. The area is not explored properly for the said scientific works, which is mainly due to the distant flora and fauna, lack of quality education and most important its location near Pak-Afghan border. The ethno medicinal traditional information are mainly limited to elder community members and local traditional health practitioners (THPs). It's a dire need of the day to document this traditional knowledge in a scientific way and conserve these medicinal plants before its loss [18]. The ethnic composition of the people is same, most of them is Khenzadghan, Sayyedan (Sayyed), Serkhani, Torkhel, and Mulan. Shetezien and Khohesthanen are migrated family which belong to Afghanistan and the primary language is Pushto. The current research project i. e. the traditional applications of the medicinal plants in Charmang, tehsil Nawagai is the first ever scientific approach to document ethno-botanical uses and report new plant species from the area. The urgent need for this kind of information is meaningful because the vegetation in the desire area of the Bajaur is extensively poor due to high Biodiversity. The Charmang area is famous due to their natural sights and beauty. Most of the plants in the area are used for medicine, timber and construction. The main objective of the present study is to file the unique information of wild medicinal plants of Charmang village, tehsil Nawagai, District Bajaur, KP, Pakistan to provide systematic basis for further examination.

\section{Materials and Methods}

\subsection{Field Interview}

The field work was conducted in Charmang Tehsil Nawagai of district Bajaur, KP, Pakistan during February 2017 - 2019 and follows the method [21, 22] to report the folk use of plants by the local peoples. A total 135 (123 males and 10 females) local Malik and Khan across different family was randomly selected for interviews, with a miner who are aware with indigence use of plants. The survey was conducted on 135 individuals from the local population, aged from 20 to 65 years and above. Informants were made for the importance medicinal plants and their knowledge was added for them. Following the Worldwide Society of Ethno biology (ISE) code of ethics the interview was conducted in sociable environment to allow the informers to response the questions logically. Ethnobotanical data were collected through verbal interviews and semi structured questionnaires [23]. Questionnaires were developed by colleague's methods [24]. Field works consisted of plant collection, photography and data documentation. The Questionnaires is consisting in two portions, in which the first portion is about personal information like age, educational level and gender of the informers, but the next portion collects treated, mode of application and their tonic uses. The interview was conducted in local language of the area (Pushto). The interview was taken in their local Hujra, of the community with reference to a place; the herbalists are interviewed at their local places. Rundown of plants were organized in order. Latin name and families were affirmed by online site: http://www.theplantlist.org (The Plant List), (The International Plant Name Index) http://www.ipni.org and Kew naming framework www.mpns.kew.org/mpnsentryway. The identified species were dried, labeled and 
attached on herbarium sheets and to deposit to the Herbarium of Abdul Wali Khan University, Mardan, Pakistan.

\subsection{Study Area and Climate Vegetation}

The geographical area of the Bajaur is about $1290 \mathrm{Km}[18$, 25], its population is more them 0.942 million. Population density is about $73 \%$ per $\mathrm{Km}^{2}$ which is highest as compare to other districts, the population growth rate about 4.33 present per annum. The Environment is mountainous to sub mountain and climate is arid and semi-arid having both winter and summer seasons (winter temperature range from 5 to 10 degree centigrade and summer temperature differs from $23^{\circ} \mathrm{C}$ to $36^{\circ} \mathrm{C}$ ) [26]. It's the smallest part (district) of the KP, which consist of two sub divisions and seven tehsils. The Charmang area is located west side of the Bajaur, which is round about $59 \mathrm{Km}$ away from headquarter namely "Khar" which is the extreme end of the Himalayan range, which creates the uncertainty and vernation in the monsoon rains [18]. The Charmang is further divided into Hilal khel, Halkai Charmang, Hasham, Kamangara Charmang, Kotakai Charmang, Shah Khanai, Sharif khana, Saida shah and Panjak. The area Charmang is located near Pak Afghan border. Charmang is mostly populated by illiterate people. The zone is situated at an elevation of 1126 m.a.s.l with E $71^{\circ} 30^{\prime}$ scope and $\mathrm{N} 34^{\circ} 41^{\prime}$ longitude. The longing zone is for the most part populated by Tarkani Pushtoons people group. The land of the Charmang valley is hilly, and fertile which take about $800 \mathrm{~mm}$ of average rainfall per year (FATA Development Statistics, 2013).

The desire area of the district Bajaur is very faraway area with no access to the basic health facilities and income resources. The people mostly dependent on the plants for most of their essentials use and people have a rich knowledge about different uses of plants for daily requirements. But the area has never been explored before for this ethno-medicinal knowledge. The deforestation rate is very high in this area the main cause of the deforestation is lake of electricity, more them $90 \%$ of the household use wood for cooking in Halki Charmang area. The other main cause of the deforestation is the operation against TTP (Tehrik-e-Taliban Pakistan) militants from since 2008. Most of the territories are farmer by calling while others are instructors and government hirelings. A low proportion of local individuals additionally acquire installment from different parts of the nation or abroad. The investigation region has constrained instruction offices, and other fundamental offices. This zone is exceptionally affected by war (started in 2009 and finish in the end of 2011) against Pak Army and TTP aggressors. Most of the people of the area use these plants as a raw material for their health. The main objective of the study to document the medicinal plants of the area and share the data with other communities through published literature. Most of the area is cultivated, while the uncultivated area is mostly arid and semi-arid, hilly, rugged and barren area. There is no good system for irrigation of the cultivated area but in some place mostly people irrigate their land by ground water, rain water and spring water.

\section{Quantitative Investigation}

\subsection{Relative Frequency of Citation (RFC)}

The area information is foreseen quantitatively using Frequency of citation (FC) and Relative Frequency of citation (RFC). The RFC was found out to exhibit the solidarity between the observers on the use of helpful plants around there. It is figured by the going with formula using equation No 1 [27].

$$
R F C=F C \div N(0<R F C<1)
$$

Where,

FC is the quantity of sources that referred to the species Relative Frequency of Citation (RFC), and $\mathrm{N}$ are the aggregate number of sources partakes in the investigation. The estimation of RFC relies upon the referring to portion of sources for that species [27-29].

\subsection{Informant Consensus Factor (ICF)}

The combination used for the calculation of plant species, by using the following formula of ICF, applying equation No $2[7,30]$.

$$
\mathrm{ICF}=\mathrm{Nur}-\mathrm{Nt} \div \mathrm{Nur}-1
$$

Where,

Nur: Stands for total number of use reports for each disease category and

$\mathrm{Nt}$ : Stands for number of taxa used for the specific category

\subsection{Use Value (UV)}

The formula used for plant calculation is UV, using equation No 3 [27, 31].

$$
U V=\Sigma U \div n
$$

Where,

$\mathrm{n}$ : refers to the total number of informants,

$\mathrm{U}$ : Stands for the number of use reports mentioned by every informant for a certain species and UV: Use value for plants carry a measurable amount for the capable point of species.

\subsection{Fidelity Level (FL)}

Fidelity level (FL) indications the ratio of informants which use and explain the plant species for exceptional purpose, using equation No 4 [32].

$$
F L=I P \div L \mathrm{u}
$$

Where, (IP) Shows the number of informants which are to be cited of a use of species for specific disease category, and ( $\mathrm{Lu}$ ) Stand for the number of informants starting the use of medicines from that plant for any sort of aliments 
category.

\section{Results and Discussion}

Demographic Characteristics of Informants

In the present research, a total 135 informants "Table 1" including indigenous people $(91.11 \%)$ and Traditional Health Practitioner's (THPs) is $(8.8 \%)$ were interviewed divided into five different age groups ranging from 20-30, 30-40, 40-50,
55-65 and 65-80 \& above years, which includes 12 herbalists and 123 people from local population. Among these individuals, larger part of men interviewed $(92.59 \%)$ which were 65-80 years of age. Based on training, indigenous learning with respect to the utilization of plants is more overwhelming among unskilled individuals (23.70\%) and this information is diminishing in exceptionally instructed class $(11.11 \%)$.

Table 1. The Demographic Data of Informants including their education, age, gender.

\begin{tabular}{|c|c|c|c|c|}
\hline S/No & Variables & Categories & Number of persons & Percentage \\
\hline \multirow{2}{*}{1} & \multirow{2}{*}{ Informant Category } & THP & 12 & $8.8 \%$ \\
\hline & & Indigenous people & 123 & $91.11 \%$ \\
\hline \multirow{2}{*}{2} & \multirow{2}{*}{ Gender } & Male & 125 & $92.59 \%$ \\
\hline & & Female & 10 & $7.40 \%$ \\
\hline \multirow{4}{*}{3} & \multirow{4}{*}{ Ages } & $20-30$ & 3 & $2.22 \%$ \\
\hline & & $30-40$ & 20 & $14.81 \%$ \\
\hline & & $55-65$ & 34 & $25.18 \%$ \\
\hline & & $65-80 \&$ above & 49 & $36.29 \%$ \\
\hline \multirow{5}{*}{4} & \multirow{5}{*}{ Education } & Illiterate & 32 & $23.70 \%$ \\
\hline & & 5 years of education & 22 & $16.29 \%$ \\
\hline & & 8 years of education & 33 & $24.44 \%$ \\
\hline & & 10 years of education & 20 & $14.81 \%$ \\
\hline & & 12 years of education & 13 & $9.62 \%$ \\
\hline
\end{tabular}

\section{Medicinal Plant Diversity}

A sum of 64 restorative plants species belonging to 39 families and 58 genera were recorded, where the given families i.e. Lamiaceae, Rhamnaceae, Asteraceae, Solanaceae and Malvaceae, having the highest numbers of species presence of 8, 4, 4, 3, and 2 species, respectively (Figure 1). The family Lamiaceae was the prevailing group of restorative plants because of the nearness of high substance of natural mixes, as carotenoids, phenolic acids, tannins, lignans, coumarins, quinones, xanthones, cucurmin, and a few other plant mixes which manages phytochemical and cell reinforcement which help to battle against various maladies, and abiotic stretch [33]. During this research, most species belongs to herbs, followed by trees and shrubs $44 \%, 37 \%$ and $19 \%$ respectively (Figure 2). This result is like the study reported in Chail valley, Tehsil Kabal District Swat Pakistan [32]. However, the antimicrobial, phytochemical and traditional studies of selected medicinal work have been published in Bajaur agency, Pakistan [25, 32, 34, 35].

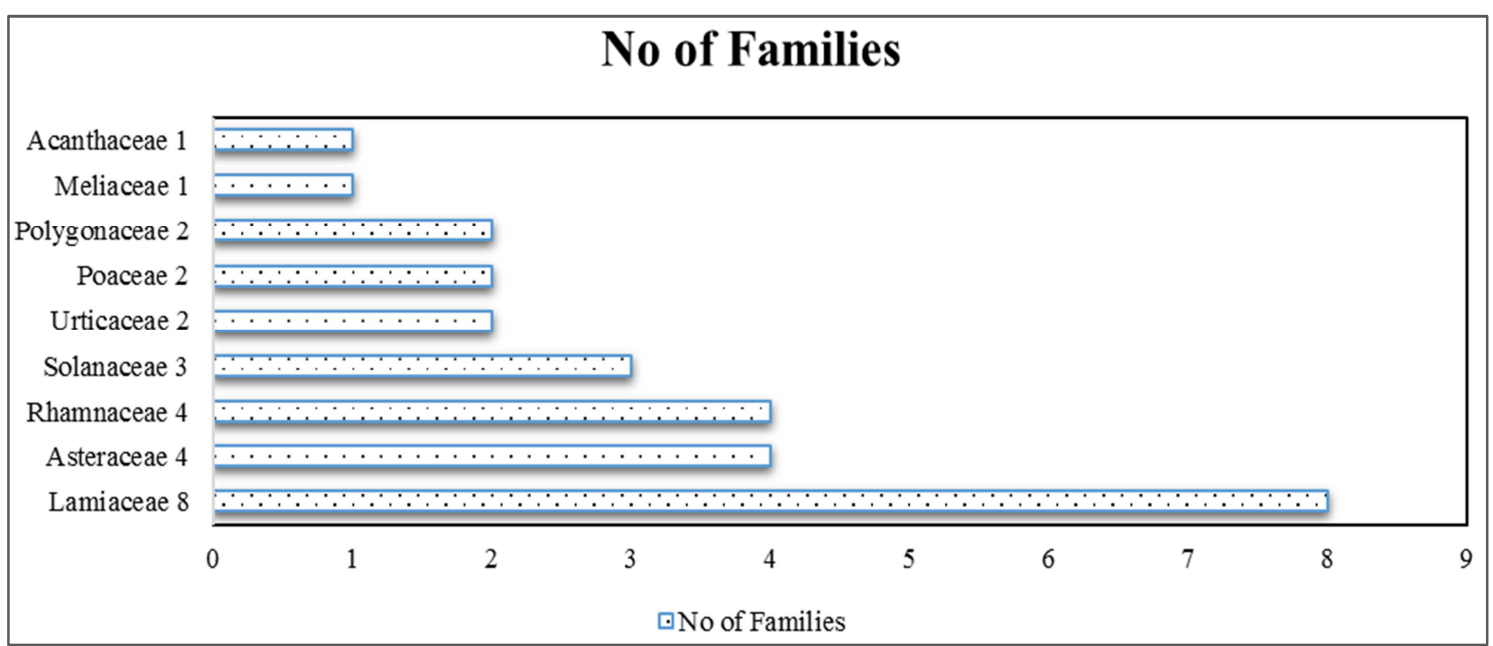

Figure 1. Density of different members of medicinal plants of various families reported from research area. Lamiaceae has the highest number, while Acanthaceae \& Meliaceae has the lowest as shown. 


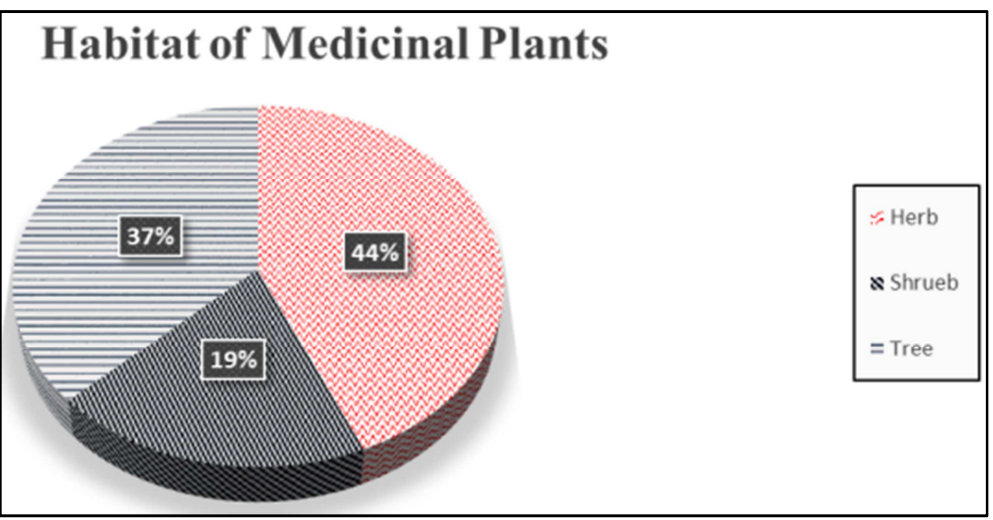

Figure 2. Percentage distribution of the habitat (types) of reported Medicinal Plants, which include herbs (44\%), shrubs (19\%) and trees (37\%).

Table 2. List of the reported Medicinal plants and their traditional applications, UV, UR, RFC and FC.

\begin{tabular}{|c|c|c|c|c|c|c|c|c|c|c|c|c|}
\hline S/No & $\begin{array}{l}\text { Plant Name } \\
\text { Voucher } \\
\text { Number }\end{array}$ & Family & $\begin{array}{l}\text { Local } \\
\text { Name }\end{array}$ & $\begin{array}{l}\text { Life } \\
\text { Form }\end{array}$ & $\begin{array}{l}\text { Plant } \\
\text { Part (s) } \\
\text { Used }\end{array}$ & $\begin{array}{l}\text { Route of } \\
\text { Administration }\end{array}$ & Diseases Treated & FC & RFC & UR & UV & Comparisons \\
\hline 01 & $\begin{array}{l}\text { Abelmoschus } \\
\text { esculentus } \\
\text { Moench. SA } \\
\text { AWKUM } 122\end{array}$ & Malvaceae & Bendhyi & Herb & Seed & Powder & Joint pain & 14 & 0.10 & 1 & 0.071 & $\begin{array}{l}1 \bullet, 2 \bullet, 3 \bullet, 4 \bullet, 5 \mathbf{\bullet}, 6 \bullet, \\
7 \bullet, 8 \bullet, 9 \bullet, 10 \bullet, 11 \bullet, \\
12 \bullet .\end{array}$ \\
\hline 02 & $\begin{array}{l}\text { Adiantum } \\
\text { capillus- } \\
\text { veneris L. SA } \\
\text { AWKUM } 133\end{array}$ & Adiantaceae & Tur panrhy & Herb & Leave & Decoction & Fever, Cough & 12 & 0.08 & 2 & 0.166 & $\begin{array}{l}1 \boldsymbol{\Delta}, 2 \bullet, 3 \bullet, 4 \bullet, 5 \mathbf{\bullet}, \\
6 \bullet, 7 \bullet, 8 \bullet, 9 \bullet, 10 \bullet, \\
11 \bullet, 12 \bullet\end{array}$ \\
\hline 03 & $\begin{array}{l}\text { Ailanthus } \\
\text { altisima } \\
\text { (Mill.) } \\
\text { Swingle. SA } \\
\text { AWKUM } 111\end{array}$ & Simaroubaceae & $\begin{array}{l}\text { Speena } \\
\text { bakhana }\end{array}$ & Tree & Seed & $\begin{array}{l}\text { Eaten in raw } \\
\text { form }\end{array}$ & $\begin{array}{l}\text { Weight loss, } \\
\text { Diabetes, Hernia }\end{array}$ & 45 & 0.33 & 3 & 0.066 & $\begin{array}{l}1 \bullet, 2 \bullet, 3 \bullet, 4 \bullet, 5 \mathbf{\bullet}, 6 \mathbf{}, \\
7 \bullet, 8 \bullet, 9 \bullet, 10 \bullet, 11 \bullet, \\
12 \bullet .\end{array}$ \\
\hline 04 & $\begin{array}{l}\text { Ajuga } \\
\text { bracteosa } \\
\text { Benth. SA } \\
\text { AWKUM } 102\end{array}$ & Lamiaceae & Ghoti & Herb & $\begin{array}{l}\text { Whole } \\
\text { plant }\end{array}$ & Powder & $\begin{array}{l}\text { Diabetes, Throat } \\
\text { pain, Blood } \\
\text { purification, } \\
\text { Alopecia }\end{array}$ & 14 & 0.10 & 4 & 0.285 & $\begin{array}{l}1 \boldsymbol{\bullet}, 2 \bullet, 3 \mathbf{\bullet}, 4 \mathbf{\Lambda}, 5 \mathbf{}, \\
6 \bullet, 7 \bullet, 8 \mathbf{\bullet}, 9 \bullet, 10 \bullet \\
11 \bullet, 12 \bullet\end{array}$ \\
\hline 05 & $\begin{array}{l}\text { Ajuga } \\
\text { parviflora } \\
\text { Benth. SA } \\
\text { AWKUM } 103\end{array}$ & Lamiaceae & Ghoti & Herb & $\begin{array}{l}\text { Whole } \\
\text { plant }\end{array}$ & Juice & $\begin{array}{l}\text { Diabetes, } \\
\text { Alopecia, } \\
\text { Androgenetic } \\
\text { alopecia }\end{array}$ & 15 & 0.11 & 4 & 0.266 & $\begin{array}{l}1 \bullet, 2 \bullet, 3 \bullet, 4 \bullet, 5 \mathbf{\bullet}, 6 \bullet, \\
7 \bullet, 8 \bullet, 9 \bullet, 10 \bullet .11 \bullet, \\
12 \bullet .\end{array}$ \\
\hline 06 & $\begin{array}{l}\text { Allium cepa L. } \\
\text { SA AWKUM } \\
115\end{array}$ & Amaryllidaceae & Pyaz & Herb & Root & Extract & Eye treatment & 33 & 0.24 & 1 & 0.030 & $\begin{array}{l}1 \bullet, 2 \bullet, 3 \bullet, 4 \bullet, 5 \mathbf{\bullet}, 6 \mathbf{}, \\
7 \bullet, 8 \bullet, 9 \bullet, 10 \bullet, 11 \bullet, \\
12 \bullet .\end{array}$ \\
\hline 07 & $\begin{array}{l}\text { Anethum sowa } \\
\text { Roxb. SA } \\
\text { AWKUM } 145\end{array}$ & Apiaceae & $\begin{array}{l}\text { Khaga } \\
\text { innaly }\end{array}$ & Herb & Seed & $\begin{array}{l}\text { Eaten in raw } \\
\text { form }\end{array}$ & Vomiting & 42 & 0.31 & 1 & 0.023 & $\begin{array}{l}1 \boldsymbol{\Delta}, 2 \bullet, 3 \bullet, 4 \bullet, 5 \bullet \\
6 \bullet, 7 \bullet, 8 \bullet, 9 \bullet, 10 \bullet \\
11 \bullet, 12 \bullet\end{array}$ \\
\hline 08 & $\begin{array}{l}\text { Berberis } \\
\text { lycium Royle. } \\
\text { SA AWKUM } \\
154\end{array}$ & Berberidaceae & Khwari & Shrub & $\begin{array}{l}\text { Root } \\
\text { bark, fruit } \\
\text { and leave }\end{array}$ & $\begin{array}{l}\text { Powder and } \\
\text { eaten in raw } \\
\text { form }\end{array}$ & $\begin{array}{l}\text { Mouth diseases, } \\
\text { Cough, Blood } \\
\text { purification, } \\
\text { Asthma, Cardia } \\
\text { Calotropisc } \\
\text { Arrhythmia, } \\
\text { Hepatitis, Healing } \\
\text { of wound }\end{array}$ & 56 & 0.41 & 9 & 0.160 & $\begin{array}{l}1 \bullet, 2 \bullet, 3 \boldsymbol{\Delta}, 4 \mathbf{\Delta}, 5 \mathbf{}, \\
6 \mathbf{\Delta}, 7 \bullet, 8 \mathbf{\bullet}, 9 \bullet, 10 \bullet \\
11 \bullet, 12 \boldsymbol{}\end{array}$ \\
\hline 09 & $\begin{array}{l}\text { Calotropis } \\
\text { procera } \\
\text { (Aiton) } \\
\text { W.T.Aiton. SA } \\
\text { AWKUM } 182\end{array}$ & Apocynaceae & Spelmai & Shrub & $\begin{array}{l}\text { Latex, } \\
\text { and leave } \\
\text { milky } \\
\text { water }\end{array}$ & $\begin{array}{l}\text { Juice, Decoction, } \\
\text { use externally } \\
\text { over skin }\end{array}$ & $\begin{array}{l}\text { anti-resistance to } \\
\text { Kangoo Virus, } \\
\text { Diabetes, Acne }\end{array}$ & 16 & 0.11 & 3 & 0.187 & $\begin{array}{l}1 \bullet, 2 \bullet, 3 \mathbf{v}, 4 \mathbf{\Delta}, 5 \mathbf{m}, \\
6 \mathbf{v}, 7 \bullet, 8 \mathbf{\bullet}, 9 \bullet, 10 \bullet \\
11 \mathbf{\bullet}, 12 \bullet\end{array}$ \\
\hline 10 & $\begin{array}{l}\text { Cedrus } \\
\text { deodara } \\
\text { (Roxb. ex } \\
\text { D.Don) } \\
\text { Loudon. SA } \\
\text { AWKUM } 166\end{array}$ & Pinaceae & sraph & Tree & $\begin{array}{l}\text { Seed and } \\
\text { bark }\end{array}$ & Oil & $\begin{array}{l}\text { Throat infection, } \\
\text { Cough }\end{array}$ & 42 & 0.31 & 2 & 0.047 & $\begin{array}{l}1 \bullet, 2 \bullet, 3 \bullet, 4 \bullet, 5 \bullet, 6 \bullet, \\
7 \bullet, 8 \bullet, 9 \bullet, 10 \bullet .11 \bullet, \\
12 \bullet\end{array}$ \\
\hline
\end{tabular}




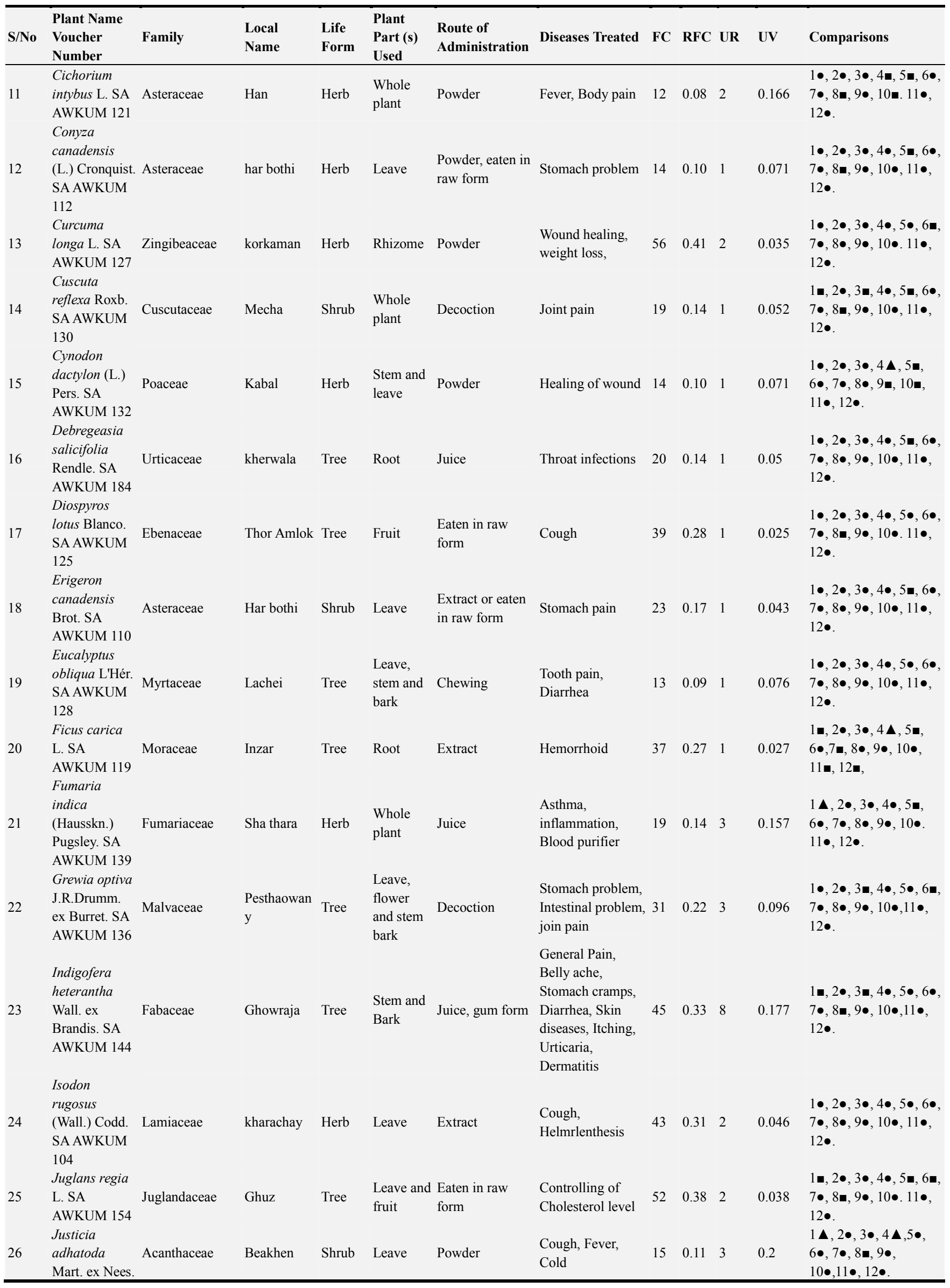




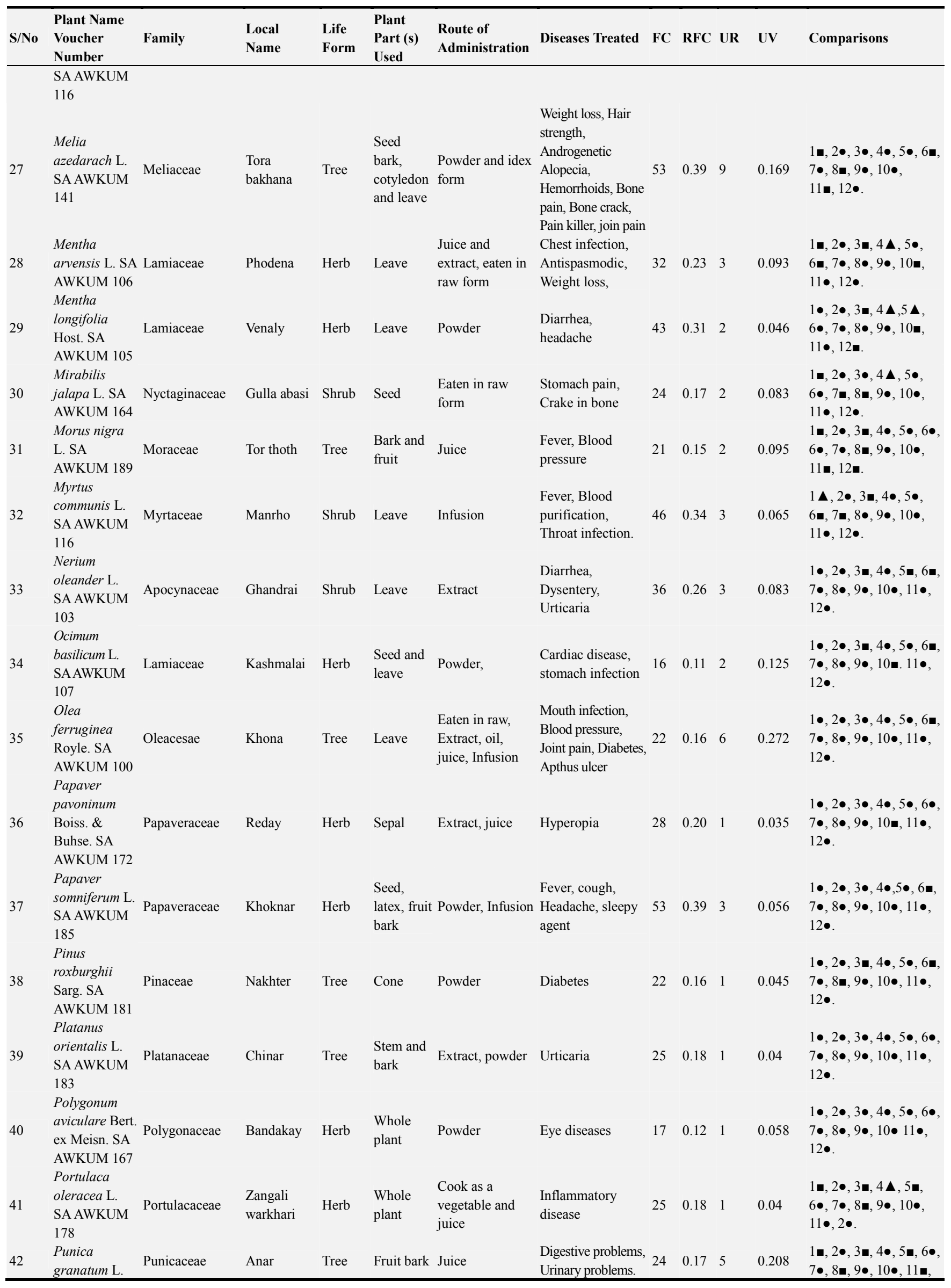




\begin{tabular}{|c|c|c|c|c|c|c|c|c|c|c|c|c|}
\hline S/No & $\begin{array}{l}\text { Plant Name } \\
\text { Voucher } \\
\text { Number }\end{array}$ & Family & $\begin{array}{l}\text { Local } \\
\text { Name }\end{array}$ & $\begin{array}{l}\text { Life } \\
\text { Form }\end{array}$ & $\begin{array}{l}\text { Plant } \\
\text { Part (s) } \\
\text { Used }\end{array}$ & $\begin{array}{l}\text { Route of } \\
\text { Administration }\end{array}$ & Diseases Treated & FC & RFC & UR & $\mathbf{U V}$ & Comparisons \\
\hline & $\begin{array}{l}\text { SA AWKUM } \\
113\end{array}$ & & & & & & $\begin{array}{l}\text { Cough, Throat } \\
\text { infection, Chest } \\
\text { pain }\end{array}$ & & & & & $12 \bullet$ \\
\hline 43 & $\begin{array}{l}\text { Ricinus } \\
\text { communis L. } \\
\text { SA AWKUM } \\
143\end{array}$ & Euphorbiacea & Arhanda & Tree & Seed & Extract & $\begin{array}{l}\text { Cough, Fever, } \\
\text { Swelling, } \\
\text { Headache }\end{array}$ & 17 & 0.12 & 4 & 0.235 & $\begin{array}{l}1 \mathbf{\Delta}, 2 \bullet, 3 \bullet, 4 \mathbf{\Lambda}, 5 \bullet \\
6 \bullet, 7 \bullet, 8 \mathbf{\bullet}, 9 \mathbf{\bullet}, 10 \bullet \\
11 \bullet, 12 \bullet\end{array}$ \\
\hline 44 & $\begin{array}{l}\text { Rosa gallica } \\
\text { L. SA } \\
\text { AWKUM } 114\end{array}$ & Rosaceae & Gullab & Shrub & Petal & $\begin{array}{l}\text { Juice Gul } \\
\text { (khand) }\end{array}$ & $\begin{array}{l}\text { Stomach pain, } \\
\text { Cough, Fever, }\end{array}$ & 39 & 0.28 & 3 & 0.076 & $\begin{array}{l}1 \bullet, 2 \bullet, 3 \bullet, 4 \bullet, 5 \bullet, 6 \bullet, \\
7 \bullet, 8 \bullet, 9 \bullet, 10 \bullet, 11 \bullet, \\
12 \bullet .\end{array}$ \\
\hline 45 & $\begin{array}{l}\text { Rumex } \\
\text { hastatus Peter. } \\
\text { SA AWKUM } \\
173\end{array}$ & Polygonaceae & Tarokay & Herb & Leave & $\begin{array}{l}\text { Juice and Eaten } \\
\text { raw form }\end{array}$ & $\begin{array}{l}\text { Blood clotting } \\
\text { agent, Diuretic, } \\
\text { Blood pressure, } \\
\text { Coagulation agent, } \\
\text { Hepatitis }\end{array}$ & 30 & 0.22 & 5 & 0.166 & $\begin{array}{l}1 \boldsymbol{\Delta}, 2 \bullet, 3 \boldsymbol{\Delta}, 4 \bullet, 5 \bullet \\
6 \bullet, 7 \bullet, 8 \bullet, 9 \bullet, 10 \bullet, \\
11 \bullet, 12 \bullet\end{array}$ \\
\hline 46 & $\begin{array}{l}\text { Salix } \\
\text { babylonica } \mathrm{L} \text {. } \\
\text { SA AWKUM } \\
175\end{array}$ & Salicacea & Walla & Tree & Leave & $\begin{array}{l}\text { Externally cover } \\
\text { the whole body }\end{array}$ & $\begin{array}{l}\text { Fever and Body } \\
\text { pain, Body tonic }\end{array}$ & 43 & 0.31 & 3 & 0.069 & $\begin{array}{l}1 \bullet, 2 \bullet, 3 \bullet, 4 \bullet, 5 \bullet, 6 \bullet, \\
7 \bullet, 8 \bullet, 9 \bullet, 10 \bullet, 11 \bullet, \\
12 \bullet .\end{array}$ \\
\hline 47 & $\begin{array}{l}\text { Salvia } \\
\text { moorcroftiana } \\
\text { Wall. ex } \\
\text { Benth. SA } \\
\text { AWKUM } 108\end{array}$ & Lamiaceae & Khor ghwag & Herb & Leave & $\begin{array}{l}\text { Externally cover } \\
\text { the infected site }\end{array}$ & $\begin{array}{l}\text { Pain killer, fever, } \\
\text { wound heeling }\end{array}$ & 34 & 0.25 & 3 & 0.088 & $\begin{array}{l}1 \boldsymbol{\Delta}, 2 \bullet, 3 \bullet, 4 \boldsymbol{\Delta}, 5 \bullet \\
6 \bullet, 7 \bullet, 8 \bullet, 9 \bullet, 10 \bullet \\
11 \bullet, 12 \bullet\end{array}$ \\
\hline 48 & $\begin{array}{l}\text { Solanum } \\
\text { nigrum Acerb. } \\
\text { ex Dunal. SA } \\
\text { AWKUM } 157\end{array}$ & Solanaceae & kamacho & Herb & $\begin{array}{l}\text { Whole } \\
\text { plant }\end{array}$ & $\begin{array}{l}\text { Juice, extract, } \\
\text { eaten in raw } \\
\text { form }\end{array}$ & $\begin{array}{l}\text { Helmrlenthesis, } \\
\text { Diabetes, } \\
\text { Dysentery, } \\
\text { Liver inflammation, } \\
\text { Kidney stone }\end{array}$ & 57 & 0.42 & 5 & 0.0877 & $\begin{array}{l}1 \mathbf{\bullet}, 2 \bullet, 3 \mathbf{\bullet}, 4 \boldsymbol{\Lambda}, 5 \bullet \\
6 \bullet, 7 \bullet, 8 \mathbf{\bullet}, 9 \mathbf{m}, 10 \mathbf{}, \\
11 \bullet, 12 \bullet\end{array}$ \\
\hline 49 & $\begin{array}{l}\text { Solanum } \\
\text { surattense } \\
\text { Burm.f. SA } \\
\text { AWKUM } 171\end{array}$ & Solanaceae & Maraghony & Herb & Fruit & Juice, Decoction & $\begin{array}{l}\text { Pain killer during } \\
\text { birth, Tonic }\end{array}$ & 11 & 0.08 & 2 & 0.181 & $\begin{array}{l}1 \boldsymbol{\Delta}, 2 \bullet, 3 \boldsymbol{\bullet}, 4 \bullet, 5 \bullet, \\
6 \bullet, 7 \bullet, 8 \bullet, 9 \bullet, 10 \bullet \\
11 \bullet, 12 \bullet\end{array}$ \\
\hline 50 & $\begin{array}{l}\text { Thlaspi } \\
\text { arvense L. SA } \\
\text { AWKUM } 127\end{array}$ & Brassicaceae & Alam & Herb & Leave & Powder & $\begin{array}{l}\text { Stomach infection, } \\
\text { Acidity, }\end{array}$ & 25 & 0.18 & 3 & 0.12 & $\begin{array}{l}1 \bullet, 2 \bullet, 3 \bullet, 4 \bullet, 5 \bullet, 6 \bullet, \\
7 \bullet, 8 \bullet, 9 \bullet, 10 \bullet, 11 \bullet, \\
12 \bullet .\end{array}$ \\
\hline 51 & $\begin{array}{l}\text { Thymus linearis } \\
\text { Benth. SA } \\
\text { AWKUM } 144\end{array}$ & Apiaceae & Spairkai & Herb & Leave & Powder & $\begin{array}{l}\text { Digestion, Asthenia, } \\
\text { Myasthenia, Colic } \\
\text { pain }\end{array}$ & 28 & 0.20 & 4 & 0.14 & $\begin{array}{l}1 \boldsymbol{\Delta}, 2 \bullet, 3 \bullet, 4 \bullet, 5 \bullet, \\
6 \bullet, 7 \bullet, 8 \bullet, 9 \bullet, \\
10 \bullet, 11 \bullet, 12 \bullet\end{array}$ \\
\hline 52 & $\begin{array}{l}\text { Trigonella } \\
\text { foenum- } \\
\text { graecum Sm. } \\
\text { SA AWKUM } \\
153\end{array}$ & Papilionaceae & Shpeshthara & Herb & $\begin{array}{l}\text { Whole } \\
\text { plant }\end{array}$ & $\begin{array}{l}\text { Extract, eaten in } \\
\text { raw form }\end{array}$ & Diabetes Mellitus & 18 & 0.13 & 1 & 0.055 & $\begin{array}{l}1 \boldsymbol{\Delta}, 2 \bullet, 3 \bullet, 4 \bullet, 5 \bullet, \\
6 \bullet, 7 \bullet, 8 \bullet, 9 \bullet, 10 \bullet, \\
11 \bullet, 12 \bullet\end{array}$ \\
\hline 53 & $\begin{array}{l}\text { Urtica dioica } \\
\text { Vell. SA } \\
\text { AWKUM } 177\end{array}$ & Urticaceae & Sezonkay & Herb & Root & Juice Extract & $\begin{array}{l}\text { Kidney stone, } \\
\text { Jaundice }\end{array}$ & 10 & 0.07 & 2 & 0.2 & $\begin{array}{l}1 \bullet, 2 \bullet, 3 \bullet, 4 \bullet, 5 \bullet, 6 \bullet, \\
7 \bullet, 8 \bullet, 9 \bullet, 10 \bullet, 11 \bullet, \\
12 \bullet .\end{array}$ \\
\hline 54 & $\begin{array}{l}\text { Viola indica } \\
\text { W.Becker. SA } \\
\text { AWKUM } 193\end{array}$ & Violaceae & Benowsha & Shrub & $\begin{array}{l}\text { Leave and } \\
\text { flower }\end{array}$ & Juice & $\begin{array}{l}\text { Fever, Flue, } \\
\text { Cough, Digestive } \\
\text { problem, Asthma }\end{array}$ & 32 & 0.23 & 5 & 0.156 & $\begin{array}{l}1 \boldsymbol{\Delta}, 2 \bullet, 3 \bullet, 4 \bullet, 5 \bullet, \\
6 \bullet, 7 \bullet, 8 \bullet, 9 \bullet, 10 \bullet, \\
11 \bullet, 12 \bullet\end{array}$ \\
\hline 55 & $\begin{array}{l}\text { Vitex negundo } \\
\text { Noronha. SA } \\
\text { AWKUM } 109\end{array}$ & Lamiaceae & Marvandai & Shrub & Leaves & Powder & $\begin{array}{l}\text { Digestive } \\
\text { problems }\end{array}$ & 23 & 0.17 & 1 & 0.043 & $\begin{array}{l}1 \boldsymbol{\Lambda}, 2 \bullet, 3 \bullet, 4 \bullet, \\
5 \bullet, 6 \bullet, 7 \bullet, 8 \bullet, 9 \bullet, \\
10 \bullet, 11 \bullet, 12 \bullet\end{array}$ \\
\hline 56 & $\begin{array}{l}\text { Withania } \\
\text { somnifera (L.) } \\
\text { Dunal. SA } \\
\text { AWKUM } 158\end{array}$ & Solanaceae & $\begin{array}{l}\text { Kher boti, } \\
\text { koti lal }\end{array}$ & Shrub & $\begin{array}{l}\text { Fruit and } \\
\text { root }\end{array}$ & Extract & Emetic, Back ache & 22 & 0.16 & 2 & 0.090 & $\begin{array}{l}1 \mathbf{\Lambda}, 2 \bullet, 3 \mathbf{m}, 4 \bullet, 5 \bullet \\
6 \bullet, 7 \bullet, 8 \mathbf{m}, 9 \bullet, 10 \bullet \\
11 \mathbf{\bullet}, 12 \bullet\end{array}$ \\
\hline 57 & $\begin{array}{l}\text { Zanthoxylum } \\
\text { armatum } \\
\text { Druce. SA } \\
\text { AWKUM } 162\end{array}$ & Rutaceae & Dambara & Tree & Fruit & $\begin{array}{l}\text { Powder, spices, } \\
\text { eaten in raw } \\
\text { form }\end{array}$ & $\begin{array}{l}\text { Tooth pain, } \\
\text { Stomach disorders }\end{array}$ & 16 & 0.11 & 2 & 0.125 & $\begin{array}{l}1 \bullet, 2 \bullet, 3 \bullet, 4 \bullet, 5 \bullet, 6 \bullet, \\
7 \bullet, 8 \bullet, 9 \bullet, 10 \bullet, 11 \bullet, \\
12 \bullet .\end{array}$ \\
\hline 58 & $\begin{array}{l}\text { Zea mays L. } \\
\text { SA AWKUM } \\
188\end{array}$ & Poaceae & Jowar & Herb & Silk & Juice & Kidney stone & 21 & 0.15 & 1 & 0.047 & $\begin{array}{l}1 \bullet, 2 \bullet, 3 \bullet, 4 \bullet, 5 \bullet, 6 \bullet, \\
7 \bullet, 8 \bullet, 9 \bullet, 10 \bullet, 11 \bullet, \\
12 \bullet .\end{array}$ \\
\hline
\end{tabular}




\begin{tabular}{|c|c|c|c|c|c|c|c|c|c|c|c|c|}
\hline S/No & $\begin{array}{l}\text { Plant Name } \\
\text { Voucher } \\
\text { Number }\end{array}$ & Family & $\begin{array}{l}\text { Local } \\
\text { Name }\end{array}$ & $\begin{array}{l}\text { Life } \\
\text { Form }\end{array}$ & $\begin{array}{l}\text { Plant } \\
\text { Part (s) } \\
\text { Used }\end{array}$ & $\begin{array}{l}\text { Route of } \\
\text { Administration }\end{array}$ & Diseases Treated & FC & RFC & UR & UV & Comparisons \\
\hline & $\begin{array}{l}\text { jujuba Lam. } \\
\text { SA AWKUM } \\
159\end{array}$ & & & & and fruit & $\begin{array}{l}\text { eaten in raw } \\
\text { form }\end{array}$ & Cough, Asthma & & & & & $\begin{array}{l}6 \bullet, 7 \bullet, 8 \bullet, 9 \bullet, 10 \bullet, \\
11 \bullet, 12 \bullet\end{array}$ \\
\hline 60 & $\begin{array}{l}\text { Ziziphus nitida } \\
\text { Roxb. SA } \\
\text { AWKUM } 161 \\
\text { Ziziphus }\end{array}$ & Rhamnaceae & $\begin{array}{l}\text { Ghwndi } \\
\text { Merkhanry }\end{array}$ & Tree & $\begin{array}{l}\text { Fruit and } \\
\text { leave }\end{array}$ & $\begin{array}{l}\text { Eaten in raw } \\
\text { form }\end{array}$ & Inflammation & 46 & 0.34 & 1 & 0.021 & $\begin{array}{l}1 \bullet, 2 \bullet, 3 \bullet, 4 \bullet, 5 \mathbf{\bullet}, 6 \bullet \text {, } \\
7 \bullet, 8 \bullet, 9 \bullet, 10 \bullet, 11 \bullet, \\
12 \bullet .\end{array}$ \\
\hline 61 & $\begin{array}{l}\text { nummularia } \\
\text { (Burm.f.) } \\
\text { Wight \& Arn. } \\
\text { SA AWKUM } \\
166\end{array}$ & Rhamnaceae & Merkhanry & Tree & $\begin{array}{l}\text { Fruit and } \\
\text { leave }\end{array}$ & $\begin{array}{l}\text { Juice, eaten in } \\
\text { raw form }\end{array}$ & $\begin{array}{l}\text { Cough, Asthma, } \\
\text { Inflammatory } \\
\text { infection }\end{array}$ & 34 & 0.25 & 3 & 0.088 & $\begin{array}{l}1 \mathbf{\bullet}, 2 \bullet, 3 \bullet, 4 \bullet, 5 \mathbf{\bullet}, 6 \bullet, \\
7 \bullet, 8 \boldsymbol{\bullet}, 9 \bullet, 10 \bullet, 11 \bullet, \\
12 \bullet .\end{array}$ \\
\hline 62 & $\begin{array}{l}\text { Quercus } \\
\text { dilatata } \text { Royle } \\
\text { SA AWKUM } \\
134\end{array}$ & Fagaceae & Serai & Tree & Root & Powder & Stomach pain & 32 & 0.23 & 1 & 0.031 & $\begin{array}{l}1 \bullet, 2 \bullet, 3 \bullet, 4 \bullet, 5 \bullet, 6 \bullet, \\
7 \bullet, 8 \bullet, 9 \bullet, 10 \bullet, 11 \bullet, \\
12 \bullet .\end{array}$ \\
\hline 63 & $\begin{array}{l}\text { Cirsium } \\
\text { arvense (L.) } \\
\text { Scop. SA } \\
\text { AWKUM } 139\end{array}$ & Asteraceae & Jahta & Herb & Seed & Powder & $\begin{array}{l}\text { Inflammatory } \\
\text { infection, Asthma } \\
\text { and abdomen pain }\end{array}$ & 45 & 0.33 & 3 & 0.066 & $\begin{array}{l}1 \bullet, 2 \bullet, 3 \bullet, 4 \bullet, 5 \bullet, 6 \bullet, \\
7 \bullet, 8 \bullet, 9 \bullet, 10 \bullet, 11 \bullet, \\
12 \bullet .\end{array}$ \\
\hline 64 & $\begin{array}{l}\text { Ziziphus } \\
\text { oxyphylla } \\
\text { Edgew. SA } \\
\text { AWKUM } 168\end{array}$ & Rhamnaceae & Welanai & Tree & $\begin{array}{l}\text { Leave and } \\
\text { root }\end{array}$ & Juice, extract & $\begin{array}{l}\text { Asthma, Cough, } \\
\text { Cardiac } \\
\text { Arrhythmia, } \\
\text { Hepatitis C. }\end{array}$ & 34 & 0.25 & 4 & 0.117 & $\begin{array}{l}1 \boldsymbol{\Delta}, 2 \bullet, 3 \bullet, 4 \boldsymbol{\Delta}, 5 \bullet \\
6 \bullet, 7 \bullet, 8 \bullet \\
9 \bullet, 10 \bullet, 11 \bullet, 12 \bullet\end{array}$ \\
\hline
\end{tabular}

FC: (Frequency of Citation), RFC: Relative Frequency of Citation, UR: Use Report. UV: Use Value. Symbols $\boldsymbol{\Delta}$ : Plants Reported with Similar Uses, $\mathbf{n}$ : Plants Reported with Dissimilar Uses, $\bullet$ : Plants Not Reported.

1: [18]; 2: [7]; 3: [18]; 4: [33]; 5: [34]; 6: [18]; 7: [16]; 8: [35]; 9: [7]; 10: [4]; 11: [36]; 12: [27].

\section{Parts of Medicinal Plants Use}

Data on medicinal plant parts used in making herbal medicine are shown in figure 3 . The most used parts of plants are leaves $(33 \%)$ flowed by seeds $(10 \%)$ fruits and whole plants $10 \%$, which is followed by root and stems $(8 \%)$. The use of leaves in herbal medicine was found commonly in several ethnomedicinal studies $[37,38]$. The regular use of leaves doesn't mean that the other plant parts having fewer active elements, it is famous that the whole plant body contains active elements. This can be clarified by the way that most informants have acquired traditional knowledge which is different from scientific learning. Leaves are more frequently use as compared with other parts because they are more in number, collection is quite easy as compared to roots, tubers and according to conservation point of view, the collection of whole plant cause the plant to be died out [26, 39].

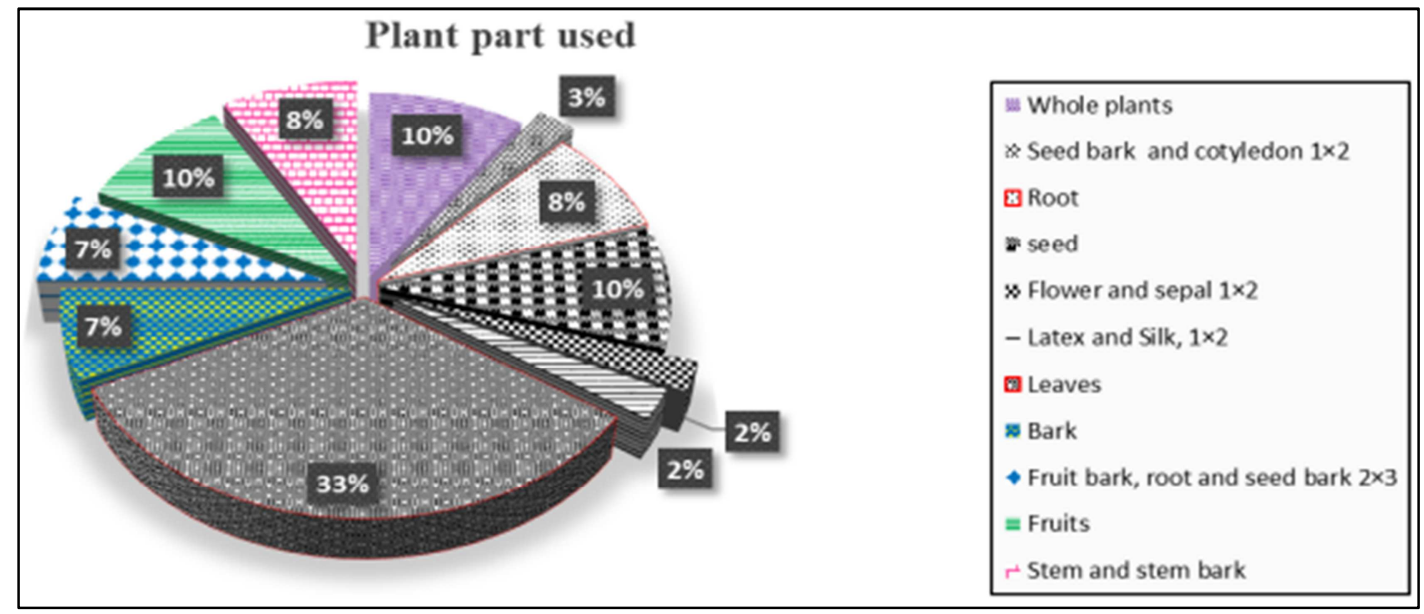

Figure 3. Graphic presentation of the percentage distribution of various plant parts used for different diseases, in which Leaves application as medicine, have been reported the highest (33\%).

\subsection{Method of Preparation and Their Applications as Herbal Medicines}

There are two modes of administration of herbal preparations i.e. (1) internal and (2) External. Internal mean decoction, infusion, teas, maceration, powder, juices etc. and External means Paste, oil, or externally cover the whole 
body. The most dominantly used preparation is the powder and juice which is 19 in numbers followed by the extract and eaten in raw form 16 from each (Figure 4). Similar results were found in the previous ethno medicinal studies conducted in different parts of the world. Most of the plants in this study are also used by mixing with honey, ghees, milk and water or cook like as vegetable. Some plants have bad test, in these causes they used to mixed it with some sweet's substance like honey, sugar etc. and eaten in raw form like Ailanthus altissima (Mill.) Swingle. Berberis lycium Royle. Mirabilis jalapa L. Solanum nigrum Acerb ex Dunal [23, 40].

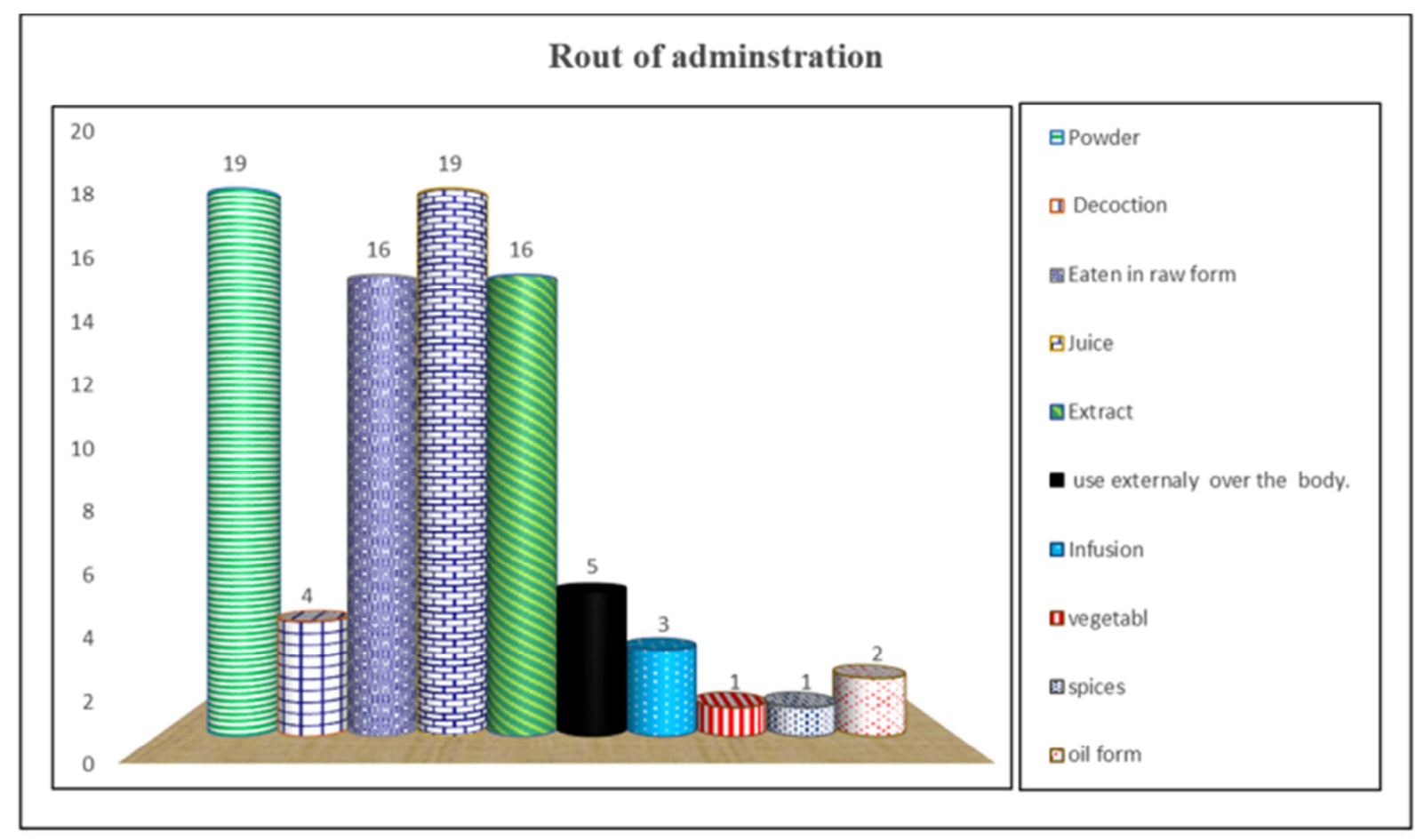

Figure 4. Percentage distribution of medicinal plants administered by local communities in various forms/products in their daily life based on data collected from inhabitants.

\subsection{Frequency of Citation (FC) of Mostly Reported Medicinal Plants and Their Uses}

In present study, the most cited plant is Solanum nigrum with 57 citations followed by Curcuma longa L. and Berberis lycium Royle 56 citations, Melia azedarach L. 53 which is followed by Ziziphus jujuba Lam, 47 citations, Myrtus communis L, Ziziphus nitida Roxb and Ziziphus nitida Roxb 46 citation, Ailanthus altissima (Mill.) Swingle and Indigofera heterantha Wall. ex Brandis 45 citation (Table 2, Figure 5). Mentha longifolia Host, Salix babylonica L and Isodon rugosus (Wall.) Codd 43 citation. The high frequency of citations of medical plants mean these plants are well known and are more frequently used by the local informers, on behalf of a source of reliability. In this study, highest number of citations is of Solanum nigrum which is used to treat Helmrlenthesis, Diabetes, Dysentery, Liver inflammation, Kidney stone Carbohydrates. We found similar result for Periploca in other ethno-medicinal surveys as well [41, 42]. Curcuma longa L, is use for weight loss and heeling of wounds. Berberis lycium Royle is used for Flavonoids, Carbohydrates, Phenols, Phlobatannins, Saponin, Cardiac Glycosides, Proteins, Volatile oils, Resins Glycosides and Terpenoids diseases, the same medicinal uses were reported in other areas of Pakistan $[4,12,28]$.

\section{Newly Reported Medicinal Plants and Their Uses}

The current study is associated with 12 published research papers, from connecting areas and from the country for similar and dissimilar uses. Current study revealed that 10 plants were reported for the first time, with new uses (Table $2 \& 3$ ). Lingering 30 plants were previously documented in earlier literature for various diseases with similar and dissimilar uses. In the proposed work we observed these new medicinal uses for one species in many diseases' categories of the country level, mention the following examples.

In present study, Ziziphus nummularia is used for treating Cough, Asthma and Inflammatory infection, Cardiac Arrhythmia, and Hepatitis C. Mentha longifolia another plant from present study is used to cure Diarrhea and headache, while previously it been reported to be used in vomiting, carminative and refrigerant. The present study is also compared with the formerly published work from Pakistan. 
Table 3. List of newly reported medicinal wild plant species from research area, which were not reported before.

\begin{tabular}{ll}
\hline S/No & Plants not reported in previous findings \\
\hline 01 & Diospyros lotus Blanco \\
02 & Erigeron canadensis Brot \\
03 & Eucalyptus obliqua L'Hér. \\
04 & Isodon rugosus (Wall.) Codd \\
05 & Platanus orientalis (L.) \\
06 & Polygonum aviculare Bert.ex Meisn. \\
07 & Rosa gallica (L.) \\
08 & Salix babylonica (L.) \\
09 & Cirsium arvense (L.) Scop \\
10 & Zea mays (L.) \\
\hline
\end{tabular}

Myrtus communis with used valve (UV) 0.065 are used frequently for Fever, Blood purification and throat infection. This plant is interesting for further phytochemical and medical studies. Based on statistical analysis following plant species are important with respect to high UV values; Ajuga bracteosa Benth (0.285) and Ajuga parviflora Benth (0.266) are the plants with high UV values, which is commonly used for diabetes blood purification and alopecia.

\section{Quantitative Analyses of Ethno Medicinal Data}

\subsection{Informant Consensus Factor (ICF)}

The value of ICF ranges from (0.130 to 0.538$)$ low to high number (Table 4). The highest and lowest values of ICF were 0.130 and 0.538 , which were reported for Gastric disorders with 24 used reports in 21 species and Skin problem respectively, which is shown in Table 4. The high (ICF) values show an extreme similarity of information among the sneaks. In the reference of highest ICF value of Cardiac disease, the similar result was found in the study reported in Thar Desert Sindh [29, 42]. Cardiac disease has been reported as well with high (ICF) values in the work reported by [9]. Now a day cardiac disease has been very common in the local community due to un stabile diets. The symptom of this disease is very common so that it can easily recognized by the traditional Hakeem, while the (ICF) values for Kidney stone (0.2) followed by Muscle and skeletal disorders (0.222) was reported.

Table 4. Disease categories of medicinal plants of Charmang, tehsil Nawagai, District Bajaur, KP, with Informant Consensus Factor (ICF).

\begin{tabular}{lllll}
\hline S/No & Disease categree & No of use report (Nur) & No of Texa (Nt) & Informant Consensus Factor (ICF) \\
\hline 01 & Blood purification & 10 & 8 & 0.222 \\
02 & Cardiac disease & 7 & 6 & 0.166 \\
03 & Diabetes & 14 & 12 & 0.153 \\
04 & Gastric disorders & 84 & 21 & 0.130 \\
05 & Helmrlenthesis & 6 & 7 & 0.142 \\
06 & Kidney stone & 4 & 5 & 0.2 \\
07 & Mouth diseases & 19 & 3 & 0.333 \\
08 & Muscle and skeletal disorders & 36 & 15 & 0.222 \\
09 & Respiratory diseases & 14 & 22 & 0.4 \\
10 & Skin problem & 7 & 0.538 \\
\hline
\end{tabular}

\subsection{Relative Frequency of Citation (RFC)}

The Relative Frequency of Citation (RFC) is used to regulate the maximum used plant species of the area with orientation to informants that have been interviewed for those plant species. The range of (RFC) was plan from high to lowest. The highest value of (RFC) was calculated 0.42 which was observed for Solanum nigrum, Berberis lycium Royle and Curcuma longa L. respectively, this status confirms that this medicinal plant species is normally found everywhere in the area and the concern people are quite familiar with its medicinal uses, mostly they are in practices for Helmrlenthesis, Diabetes, Dysentery, Liver inflammation, Kidney stone, Mouth diseases, Cough, Blood purifier, Asthma, Cardia, Calotropisc Arrhythmia, Hepatitis, Healing of wound, and weight loss. The most frequently used medicinal plants species in the study area are Solanum nigrum (0.42), Berberis lycium Royle (0.41), Curcuma longa L. (0.41), Papaver somniferum (0.39), Melia azedarach (0.39) and Juglans regia (0.38) respectively (Table 2).

\subsection{Use Value (UV)}

UV (use value) is a quantitative method that shows the relative importance of a plant species or plant family among the population. In the present study, the UV was calculated and ranges from highest to lowest species. The highest VU in term of numbering sequence was reported in Solanum nigrum (0.42) followed by Berberis lycium Royle (0.41), Curcuma longa L. (0.41), Papaver somniferum (0.39), Melia azedarach (0.39) and Juglans regia (0.38) respectively. The high UV values show that these specific plant species are the most recommended and well identified plant for the local people, and its specific uses. 


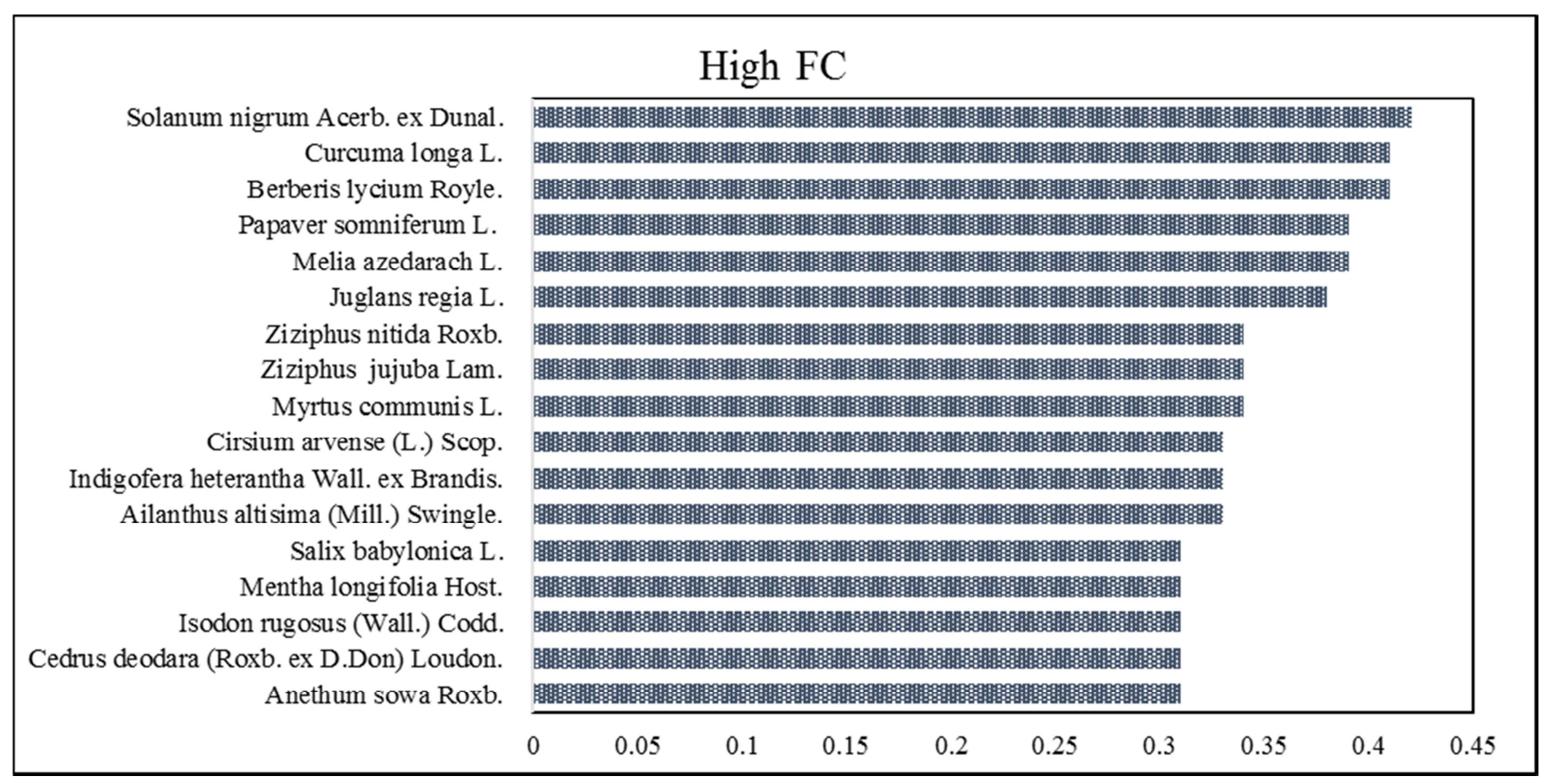

Figure 5. Graphic illustration of the Frequency of Citation (FC) of various reported medicinal plants from research area.

However, the plants species with lowest UV indicate that these plants are very rare in the area due to the medicinal knowledge and concern information by the local community.

\subsection{Future Effect of the Study and Recommendation}

The examination will give a feeling of social and financial obligation between the network, to ensure the nearby verdure. This acknowledged data will likewise empower the neighborhood network to pull in tourism in the zone by rationing its common magnificence, which will expand the financial achievement and prosperity of the rustic network. The commitment of the nearby network will help in keeping the flower decent variety and to advance exchange and tourism. In any case, then specialists occupied with the arrangement making could deliver the issues identified with the floristic structure and preservation. The pharmacological and nourishment assembling could simply abuse the nearby therapeutic verdure, which could be utilized for the general well-being and financial inspire of the region. This examination will additionally demonstrate the essential data of neighborhood individuals and relocated people groups of the territory, it's likewise pulls in the nearby government to give fundamental wellbeing resources and training to the said network.

\section{Conclusion}

Th current investigation reports the important of the medicinal plants used in ethnomedicinal practices at Charmang district Bajaur, KP, Pakistan. Indigenous communities of the desire region usually rely on local flora for their medicinal practices and the traditional knowledge is in the protection of elder people. The study highlights that due to modernization, the younger generation does not take interest in the application of medicinal plants which is present in this local flora. This area is highly affected by "TTP War" which effect the local flora [43]. In this research area a total of 64 medicinal plants have been recorded, in which most of the plants belongs to family Lamiaceae followed by Asteraceae (Figure 1). Furthermore, the current investigation reported that $D$. lotus, E. canadensis, Eucalyptus obliqua, Isodon rugosus, Platanus orientalis, Polygonum aviculare Rosa gallica, Salix babylonica, Cirsium arvense and Zea mays wild plants which are not reported in previous findings have been reported for the first time with new uses for various diseases, which is enlist in (Table 3). It was also found during interview of local traditional healers and local respondents of the study area that most of the peoples use these wild plants species for fever, stomach pain, kidney stone and other different illness (table 2). On the base of the studied area, most of the diseases which treated by these plants are very common in daily life, such is cough, fever and infection etc (table 4). According to the survey result, most of the wild plants species is used for various diseases such as diabetes, asthma, hepatitis fever and different infections (table $2 \& 4$ ). According to the survey mostly peoples of the Charmang use these plants for primary health care. Most the use these plant as a vegetable (Sagh). According to the interviewed reports the most frequently use plants is Solanum nigrum and curcuma longa etc as shown in figure 5. Mostly herbs were found to be the most common life form (44\%) as compare to tree (37\%) and shrub (19\%) in different preparations.

Furthermore, these areas are near the Pak afghan border, so there is very highly biodiversity and natural vegetation, but there is very highly loss of the natural vegetation. In these studies, the result show that all the plants species ware reported from the local communities of the desire area. In 
these research articles the (Table 4) shows that ten plants species ware informed in traditional herbal method for the first time. This study can donate well in the protection of world traditional heritage as well as the collection of valuable knowledge to document a local record and improve the existing practices. Meanwhile, it may contribute in writing the pharmacopeia in a better strategy, which is still a question subject. The proposed results show that the cultivators and local community have an energetic and significant understanding showed by the assortment of various species used to treat few disorders, which is considered a worthy hotspot for social event. The Ethno medicinal and pharmacological data in the region comparatively show fundamentals that how the societies and civilizations affect the entire basin's herbal knowledge in term of science promotion in various field, including medicine, pharmacy, biochemistry and biology in general.

Charmang Valley is famous due to its unique landscape and location, which consists of hilly areas and good forest. Most of the local peoples collect these plants from their local habitat and generally use it for commercial and domestic uses without conservation policy. The over grazing and cause the reduction of natural habitats. It's necessary to study the pharmacological, phytochemical, antimicrobial, toxicological activity of the remaining reported medicinally important plants species to make a better understanding about the safety and efficacy of the recommended dosage. It's also necessary for preservation to aware the local people about the importance of traditional herbal knowledge and motivate them regarding the sustainable use of the local flora.

\section{Acknowledgements}

The author is very grateful to the local communities and Traditional Health Practitioners (THPs) for being sharing their indentions knowledge during the study. We are also thankful to Dr. Abdur Rauf, Dr. Gul Jan and to my brothers Sayyed Hamdullah and Syed Hidayatullah for support throughout my research work during plants collection survey. I am also thankful to the Herbarium of Abdul Wali Khan University, Garden Campus, Mardan, KP, Pakistan for their help and support.

\section{References}

[1] Amjad, M. S, Ethnobotanical profiling and floristic diversity of Bana Valley, Kotli (Azad Jammu and Kashmir), Pakistan. Asian Pacific Journal of Tropical Biomedicine, 2015. 5(4): p. 292-299.

[2] Amjad, M. S, et al, Ethnobotanical profiling of the medicinal flora of Kotli, Azad Jammu and Kashmir, Pakistan: Empirical reflections on multinomial logit specifications. Asian Pacific journal of tropical medicine, 2017. 10(5): p. 503-514.

[3] Mbaveng, A. T, Q. Zhao, and V. Kuete, Harmful and protective effects of phenolic compounds from African medicinal plants, in Toxicological Survey of African Medicinal Plants. 2014, Elsevier. p. 577-609.
[4] Pieroni, A, et al, Traditional phytotherapy and trans-cultural pharmacy among Turkish migrants living in Cologne, Germany. Journal of ethnopharmacology, 2005. 102(1): p. 6988 .

[5] Khaled-Khodja, N, L. Boulekbache-Makhlouf, and K. Madani, Phytochemical screening of antioxidant and antibacterial activities of methanolic extracts of some Lamiaceae. Industrial crops and products, 2014. 61: p. 41-48.

[6] Mahmood, A, et al, Ethno medicinal survey of plants from district Bhimber Azad Jammu and Kashmir, Pakistan. Journal of Medicinal Plants Research, 2011. 5(11): p. 2348-2360.

[7] Iqbal, T, et al, ETHNOMEDICINAL STUDY OF FLORA OF DISTRICT KARAK, KHYBER PAKHTUNKHWA, PAKISTAN. International Journal, 2014. 2(9): p. 88-93.

[8] Malik, K, et al, Traditional plant based medicines used to treat musculoskeletal disorders in Northern Pakistan. European Journal of Integrative Medicine, 2018. 19: p. 17-64.

[9] R Vasanthi, H, N. ShriShriMal, and D. K Das, Phytochemicals from plants to combat cardiovascular disease. Current medicinal chemistry, 2012. 19(14): p. 2242-2251.

[10] Edwards, S, S. Nebel, and M. Heinrich, Questionnaire surveys: Methodological and epistemological problems for field-based ethnopharmacologists. Journal of Ethnopharmacology, 2005. 100(1-2): p. 30-36.

[11] Pieroni, A. and C. L. Quave, Traditional pharmacopoeias and medicines among Albanians and Italians in southern Italy: a comparison. Journal of Ethnopharmacology, 2005. 101(1-3): p. 258-270.

[12] Hussain, K, A. Shahazad, and S. Zia-ul-Hussnain, An ethnobotanical survey of important wild medicinal plants of Hattar district Haripur, Pakistan. Ethnobotanical leaflets, 2008. 2008(1): p. 5 .

[13] Amjad, M. S. and M. Arshad, Ethnobotanical inventory and medicinal uses of some important woody plant species of Kotli, Azad Kashmir, Pakistan. Asian Pacific Journal of Tropical Biomedicine, 2014. 4(12): p. 952-958.

[14] Adnan, M, et al, Ethnomedicine use in the war affected region of northwest Pakistan. Journal of Ethnobiology and Ethnomedicine, 2014. 10(1): p. 16.

[15] Shuaib, M, et al, Ethnobotanical and ecological assessment of plant resources at District Dir, Tehsil Timergara, Khyber Pakhtunkhwa, Pakistan. Acta Ecologica Sinica, 2018.

[16] Aziz, M. A, et al, Traditional phytomedicines for gynecological problems used by tribal communities of Mohmand Agency near the Pak-Afghan border area. Revista Brasileira de Farmacognosia, 2018.

[17] Aziz, M. A, et al, Traditional uses of medicinal plants practiced by the indigenous communities at Mohmand Agency, FATA, Pakistan. Journal of ethnobiology and ethnomedicine, 2018. 14(1): p. 2.

[18] Aziz, M. A, et al, Traditional uses of medicinal plants reported by the indigenous communities and local herbal practitioners of Bajaur Agency, Federally Administrated Tribal Areas, Pakistan. Journal of ethnopharmacology, 2017. 198: p. 268-281.

[19] Akhtar, N, et al, Diversity and use of ethno-medicinal plants in the region of Swat, North Pakistan. Journal of ethnobiology and ethnomedicine, 2013. 9(1): p. 25. 
[20] Cox, P. A, Will tribal knowledge survive the millennium? Science, 2000. 287(5450): p. 44-45.

[21] Hussain, F, A. Khaliq, and M. Durrani. Ethnobotanical studies on some plants of Dabargai Hills. Swat. in Proceedings of first training workshop on Ethnobotany and its application to conservation. 1996.

[22] Hussain, F, K. Khaliq, and M. Durrani. Ethnobotanical studies on some plants of Dabargia Hills, Swat: 207-215. in Proceedings of first Training Workshop on Ethno botany and its Application to Conservation. NARC, Islamabad. 1996.

[23] Rashid, H. A. and I. Simmonds, Eddy-zonal flow interactions associated with the Southern Hemisphere annular mode: Results from NCEP-DOE reanalysis and a quasi-linear model. Journal of the atmospheric sciences, 2004. 61(8): p. 873-888.

[24] Galabuzi, C, et al, Traditional medicine as an alternative form of health care system: a preliminary case study of Nangabo sub-county, central Uganda. African Journal of Traditional, Complementary and Alternative Medicines, 2010. 7(1).

[25] Ahmad, I, M. Ibrar, and N. Ali, Ethnobotanical study of Tehsil Kabal, Swat District, KPK, Pakistan. Journal of Botany, 2011. 2011.

[26] Rehman, S, L. M. Al-Hadhrami, and M. M. Alam, Pumped hydro energy storage system: A technological review. Renewable and Sustainable Energy Reviews, 2015. 44: p. 586-598.

[27] Wali, R, et al, A quantitative medico-botanical expedition of Fairy Meadows National Park, Diamir, Gilgit Baltistan, Pakistan. bioRxiv, 2019: p. 507848.

[28] Nandagopal, S. and B. R. Kumari, Phytochemical and antibacterial studies of Chicory (Cichorium intybus L.)-A multipurpose medicinal plant. Advances in Biological Research, 2007. 1(1-2): p. 17-21.

[29] Yaseen, G, et al, Ethnobotany of medicinal plants in the Thar Desert (Sindh) of Pakistan. Journal of ethnopharmacology, 2015. 163: p. 43-59.

[30] Calixto, J. B, Twenty-five years of research on medicinal plants in Latin America: a personal view. Journal of ethnopharmacology, 2005. 100(1-2): p. 131-134.

[31] Heinrich, M, et al, Ethnopharmacological field studies: a critical assessment of their conceptual basis and methods. Journal of Ethnopharmacology, 2009. 124(1): p. 1-17.

[32] Ahmad, M, et al, An Ethnobotanical study of Medicinal Plants in high mountainous region of Chail valley (District SwatPakistan). Journal of ethnobiology and ethnomedicine, 2014. 10(1): p. 36.
[33] Kadir, M. F, M. S. B. Sayeed, and M. Mia, Ethnopharmacological survey of medicinal plants used by indigenous and tribal people in Rangamati, Bangladesh. Journal of ethnopharmacology, 2012. 144(3): p. 627-637.

[34] Aziz, M. A, et al, Traditional uses of medicinal plants used by Indigenous communities for veterinary practices at Bajaur Agency, Pakistan. Journal of ethnobiology and ethnomedicine, 2018. 14(1): p. 11.

[35] Ibrar, M, et al, Quantitative ethnobotanical survey of medicinal flora thriving in Malakand Pass Hills, Khyber Pakhtunkhwa, Pakistan. Journal of ethnopharmacology, 2015. 169: p. 335-346.

[36] Khan, M. and S. Musharaf, Ethnomedicinal and conservation status of plant species in Tehsil Takht Bhai, District Mardan, Pakistan. International Letters of Natural Sciences, 2015. 37.

[37] Kayani, S, et al, Ethnobotany of medicinal plants among the communities of Alpine and Sub-alpine regions of Pakistan. Journal of ethnopharmacology, 2015. 164: p. 186-202.

[38] Jasvinder, A, D. Furst, and A. Bharat, update of the 2008 American College of Rheumatology recommendations for the use of disease-modifying antirheumatic drugs and biologic agents in the treatment of rheumatoid arthritis. Arthritis Care Res, 2012. 64: p. 625-39.

[39] Ribeiro, J, et al, Identification of nanominerals and nanoparticles in burning coal waste piles from Portugal. Science of the Total Environment, 2010. 408(23): p. 60326041 .

[40] Bano, A, et al, Quantitative ethnomedicinal study of plants used in the skardu valley at high altitude of KarakoramHimalayan range, Pakistan. Journal of ethnobiology and ethnomedicine, 2014. 10(1): p. 43.

[41] Rashid, S, et al, Ethnobotanical survey of medicinally important shrubs and trees of Himalayan region of Azad Jammu and Kashmir, Pakistan. Journal of ethnopharmacology, 2015. 166: p. 340-351.

[42] Mushtaq, R, et al, Ethnobotanical survey of medicinally important shrubs and trees of Himalayan region of Azad Jammu and Kashmir, Pakistan. Journal of ethnopharmacology, 2015 .

[43] Aziz, M. A, et al, Cross-Cultural Analysis of Medicinal Plants commonly used in Ethnoveterinary Practices at South Waziristan Agency and Bajaur Agency, Federally Administrated Tribal Areas (FATA), Pakistan. Journal of ethnopharmacology, 2018. 210: p. 443-468. 